\title{
MINOR (NR4A3) Overexpression in Mouse Skeletal MuscleEnhances Insulin Action
}

Wei Zhang, John Garvey W, Nanlan Luo, W Timothy Garvey and Yuchang Fu

${ }^{1}$ Department of Nutrition Sciences, University of Alabama at Birmingham, Birmingham AL 35294-3360, USA

*Corresponding author: Yuchang Fu, PhD, Department of Nutrition Sciences, The University of Alabama at Birmingham, 1825 University Boulevard, Birmingham, AL 35294-0012, USA, Tel: 205-996-4013; Fax: 205-996-5896; E-mail:yfu@uab.edu

Received date: February 3, 2013; Accepted date: February 19, 2014; Published date: February 24, 2014

Copyright: $\complement^{2014}$ Zhang W, et al. This is an open-access article distributed under the terms of the Creative Commons Attribution License, which permits unrestricted use, distribution, and reproduction in any medium, provided the original author and source are credited.

\begin{abstract}
MINOR (Mitogen-Inducible Nuclear Orphan Receptor) is one member of the NR4A3 nuclear orphan receptor family which are immediate early gene products involved in neuroendocrine regulation, neural differentiation, liver regeneration, cell apoptosis, and mitogenic and inflammatory stimulation in different cell types. We have found that MINOR can modulate insulin action and the glucose transport system in 3T3-L1 adipocytes; however, MINOR is highly expressed in skeletal muscle and its function in vivo is not well understood.
\end{abstract}

To determine the role of MINOR in vivo, we have generated a mouse model that has the MINOR gene specifically expressed in the skeletal muscle using a muscle creatine kinase (MCK) promoter, and investigated whether the gene functions of MINOR would be linked to insulin action in vivo since skeletal muscle is one of the primary target tissues for insulin action.

We demonstrate that these MCK-MINOR transgenic mice have reduced body weight due to a reduction of fat mass inside the body. Mice with MINOR overexpression also have improved insulin and glucose tolerances, reduced plasma levels of triglyceride, cholesterol and free fatty acid as well as enhanced expression of genes which are related to insulin action and its signaling pathways. Thus, MINOR functions in skeletal muscle act to improve insulin sensitivity and glucose intolerances and regulate insulin action and lipid and energy expenditure process.

Keywords: NR4A Orphan Nuclear Receptors; Muscle; Insulin and glucose; Intolerances; Obesity; Diabetes

\section{Introduction}

Type 2 diabetes is a disease caused by defects in insulin secretion combined with the presence of insulin resistance in peripheral target tissues which mainly include skeletal muscle, adipose tissue, and liver. To better understand the molecular defects responsible for human insulin resistance, we previously assessed differential gene expression in human skeletal muscle biopsies from insulin-sensitive and -resistant individuals using cDNA microarray technology [1]. These studies identified one of the differentially expressed genes that are members of the NR4A (NGFI-B) family of orphan nuclear receptors within the greater steroid/thyroid receptor superfamily, namely, MINOR (Mitogen-Inducible Nuclear Orphan Receptor).

MINOR (also known as NR4A3, NOR-1, TEC, and CHN) was originally identified as a protein induced in primary cultures of rat embryonic forebrain neurons undergoing apoptosis [2]. Based on homology within the DNA binding domain, there are two additional members of the NR4A (NGFI-B) family of orphan nuclear receptors, Nurr1 (also known as NR4A2, TINUR, and NOT) and Nur77 (also known as, NR4A1, NGFI-B, and TR3). These NR4A/NGFI-B proteins are immediate early response gene products that are involved in neuroendocrine regulation, neural differentiation, liver regeneration, cell apoptosis, and mitogenic and inflammatory stimulation in different cell types [3-8].
Based on our unpublished data, MINOR is highly expressed in human skeletal muscle but almost undetectable in other tissues. We have recently overexpressed MINOR in a mouse skeletal muscle cell line, $\mathrm{C} 2 \mathrm{C} 12$, and found that overexpression of MINOR in these cells can lead to a significant increase in glucose transport activity in the presence of insulin and insulin-mediated AKT phosphorylation was also increased by the MINOR overexpression in these cells [9]. These results and our previous data from adipocytes [10] confirmed that MINOR is able to enhance insulin sensitivity in these insulin target cells.

To further investigate whether MINOR has the same effects on increasing insulin sensitivity in vivo, we have generated a mouse model, MCK-MINOR, which has the MINOR genespecifically expressed in the skeletal muscle using a muscle creatine kinase (MCK) promoter. Our current studies have shown that MINOR overexpression in skeletal muscle results significant improved systemic insulin sensitivity and glucose tolerance in vivo and MINOR also enhances some key components of insulin signaling pathway for insulin action in skeletal muscle, suggesting that MINOR as a novel modulator to function in skeletal muscle for modulating insulin actions.

\section{Materials and Methods}

\section{Experimental mice}

Fusion cDNAs, containing the full length MINOR coding sequence and a V5 epitope tag, were cloned into a MCK enhancer/promoter 
cassette vector (a kind gift from Drs. Morris Birnbaum and Ron Kahn). Before microinjection into mouse embryos, the gene fragments $(9.5 \mathrm{~kb})$ were purified. Transgenic animals were created by injections of the appropriate DNA fragments into the pronucleus of inbred FVB single cell embryos. Three independent lines of MINOR transgenic mice were obtained and identified by Southern blotting or by PCR. Tissue specific expression was verified by RT-PCR with one primer designed in the V5 sequences and another located in the MINOR sequences. Animals were housed in colony cages and maintained on a 12 hours light/ 12 hours dark cycle. The animal care and procedures were approved by the Animal Resources Program (ARP) of the University of Alabama at Birmingham.

To investigate the metabolism of these MINOR transgenic mice, a regular diet or a high fat diet ( $60 \% \mathrm{kcal} \%$ fat) from the Research Diets Company (New Brunswick, NJ) was fed to control and transgenic mice from 4 weeks to 36 weeks for measuring mouse body weight and analyzing glucose metabolism and gene expression in skeletal muscle.

\section{Immunoblots}

Soleus muscles were carefully dissected from mice and quickly frozen in liquid nitrogen. Muscle tissues were homogenized with tissue lysis buffer $(1 \times$ PBS, $1 \%$ Nonidet P- $40,0.5 \%$ sodium deoxycholate, $0.1 \%$ SDS) containing freshly added protease inhibitor cocktail (Sigma). Twenty-five $\mu \mathrm{g}$ of protein per lane and known molecular weight markers from Bio-Rad (Hercules, CA) were separated by SDSpolyacrylamide gel electrophoresis. Proteins were electrophoretically transferred onto nitrocellulose membranes and incubated overnight at $4^{\circ} \mathrm{C}$ with blocking solution ( $5 \%$ nonfat milk in TBS). The blocked membranes were incubated with gene specific antibodies, and then the horseradish peroxidase (HRP)-conjugated second antibody for 1 hour for each at room temperature, and washed three times for each with TBS buffer containing $0.1 \%$ Tween 20 for 15 minutes at room temperature with shaking. Immunodetection analyses were accomplished using the Enhance Chemiluminescence Kit (NEN Life Science Products, Boston, MA).

\section{Glucose and insulin tolerance tests}

Glucose tolerance tests were performed in mice after 12 hours fast. Insulin tolerance tests were performed in mice after 6 hours fast. Glucose and insulin solutions were injected into peritoneal cavity at the dose of $1.0 \mathrm{~g} / \mathrm{kg}$ and $1.0 \mathrm{U} / \mathrm{kg}$, respectively. Blood was collected via tail vein at different time points, and glucose levels were measured by the use of a glucometer (Precision). Serum lipid concentrations were determined by established assays as previously described [11].

\section{Glucose transport activity assays}

Glucose uptake assays in soleus muscle strips were performed as described before [12]. For measurement of glucose transport activity, muscle strips were treated with $100 \mathrm{nM}$ insulin for one hour then the muscle strips were treaded with $1 \mathrm{~N} \mathrm{NaOH}$ at $70^{\circ} \mathrm{C}$ for $5 \mathrm{~min}$; the aliquots of the supernatant were centrifuged and added to the scintillation mixtures and then counted for the isotope activities. In these experiments, the distribution space of radiolabeled L-glucose was used to correct for nonspecific carryover of radioactivity with the cells and uptake of hexose by simple diffusion.

\section{Statistics}

Experimental results are shown as mean \pm SE. Statistical analyses were performed by unpaired Students' $t$-test assuming unequal variance unless otherwise indicated. Significance was defined as $p<0.05$.
A

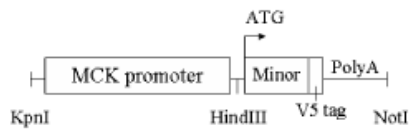

C

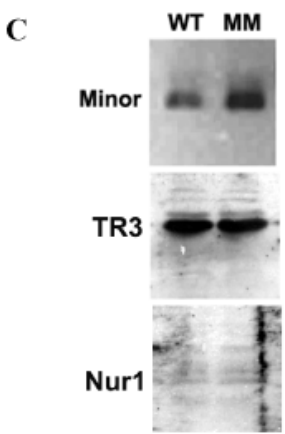

B

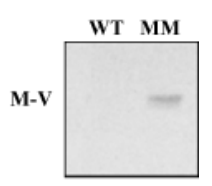

Figure 1: Skeletal muscle tissue-specific expression of MINOR. (A) Skeletal muscle specific expression of MINOR gene was achieved by a $6.5 \mathrm{~kb}$ of muscle creatine kinase (MCK) promoter/enhancer sequences. Hind III restriction enzyme site was used to insert the fusion gene (MINOR coding sequences plus a V5 tag) into the promoter/enhancer vector; the DNA fragment $(9.6 \mathrm{~kb})$ was digested with Kpn I and Not I enzyme sites and purified before microinjected into mouse embryos. (B) MINOR transgene expression was specifically detected in aged 12 weeks of mouse skeletal muscle by a RT-PCR assay with 5' primer (5'-ATA TCC AAG CCT TAG CCT GCC TGT-3') targeting to MINOR coding sequences and 3' primer (5'-TCC AAA CTC ATT ACT AAC CGG TAC GCG-3') targeting to the V5 tag sequences (M-V indicated the MINOR-V5 PCR fragment, $336 \mathrm{bp}$ ). WT and MM refer to wild type and MCK-MINOR transgenic mice, respectively. (C) MINOR (NR4A3) and the two other members of the NR4A nuclear family, TR3 (NR4A1) and Nurr1 (NR4A2) were examined at 16 weeks of age using Western blot analyses with specific antibodies against to these nuclear proteins in the skeletal muscle tissues either from control wild type (WT) or MCK-MINOR (MM) transgenic mice. Results represented the mean \pm SE from three separate experiments.

\section{Results}

\section{Generation of MINOR skeletal muscle transgenic mice}

Overexpression of MINOR was selectively performed in mouse skeletal muscle using the MCK promoter. Skeletal muscle specific expression of MINOR gene was achieved by ligating 6.5 kilobases (kb) of the MCK promoter/enhancer to the MINOR coding sequences (Figure 1A). MINOR transgene expression was restricted to skeletal muscle (Figure 1B) and its expression level was 2.35 -fold $(p<0.05)$ when comparing to endogenous gene expression in the control wild 
type mice (Figure 1C). The tissue specificity and high efficiency of MINOR transgene activity were not observed in other metabolic tissues, such as, liver and adipose tissue. Furthermore, other two members of the NR4A nuclear family, TR3 (NR4A1) and Nurr1 (NR4A2) expression levels showed no significant chances in skeletal muscle due to the overexpression of MINOR in this tissue (Figure 1C), suggesting that there are no functional compensations occurred among MINOR and other two nuclear receptor family members in skeletal muscle.

\section{Reduction of whole body weight in transgenic mice}

Although there was no gross apparent abnormality in reproduction, growth, and development of MCK-MINOR transgenic mice under standard laboratory conditions in either sex, we observed that transgenic mice exhibited a reduction $(15 \%)$ in total body weight between 5 to 11 weeks on high fat diet when compared to control wild type animals despite normal axial growth (Figure 2A), suggesting that overexpression of MINOR in skeletal muscle might influence adiposity in these animals. To assess whether this reduction in total body weight gain in MCK-MINOR transgenic mice is related to local and systemic alterations in adiposity, we dissected these mice for analyzing abdominal fat pads and also performed dual energy X-ray absorption (DEXA) analyses for total body composition. Both of dissection and DEXA analyses of MCK-MINOR mice demonstrated significantly reduced total body adipose mass in these mice when compared to control wild type animals (Figure $2 \mathrm{~B}$ ), suggesting that overexpression of MINOR in skeletal muscle can significantly affect adiposity, especially adipose tissue accumulation in the body under high fat diet condition.

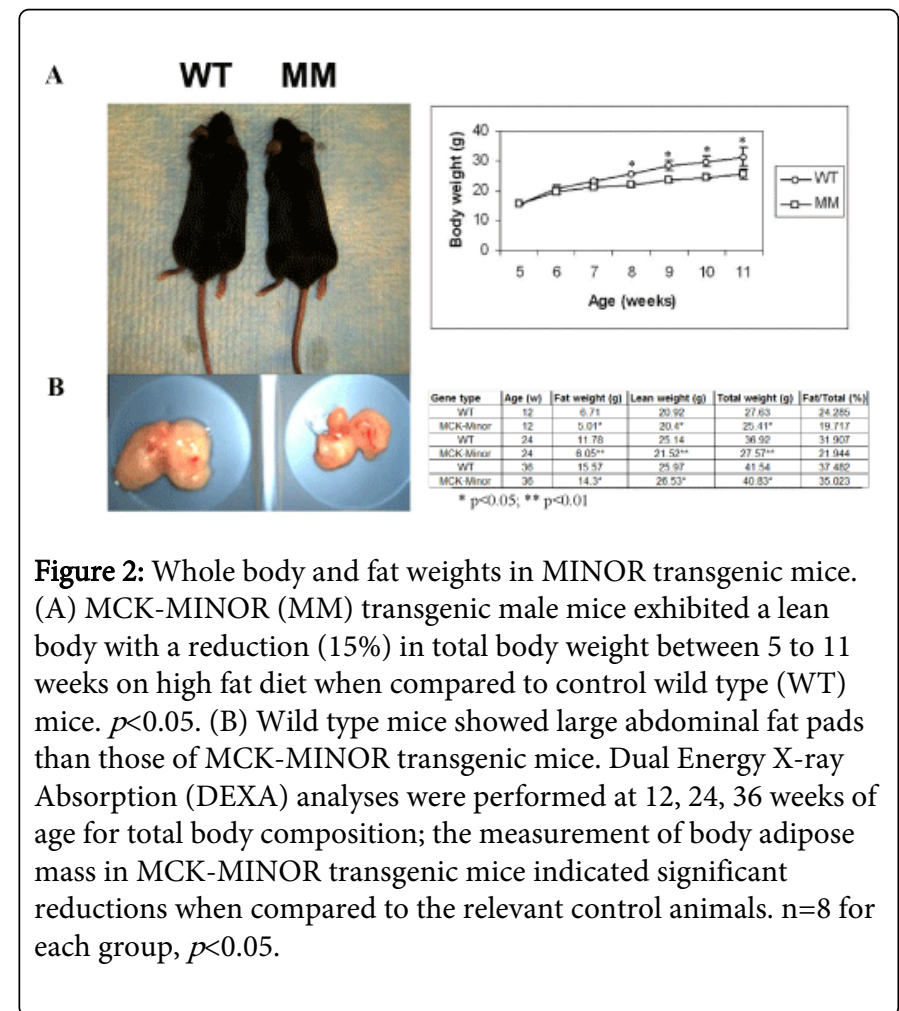

\section{Improved glucose and insulin tolerances in transgenic mice}

Next, to further investigate systemic insulin sensitivity in vivo, we performed Glucose Tolerance Test (GTT) and Insulin Tolerance Test (ITT) in MCK-MINOR mice and control wild type animals. Glucose tolerance tests revealed a higher degree of hyperglycemia in wild type animals through the experiments when compared to MCK-MINOR mice either on regular diet or high fat diet (Figure 3A). As shown in Figure $3 \mathrm{~B}$, the increase in insulin sensitivity in insulin tolerance tests was most dramatic in MCK-MINOR mice on either regular diet or high fat diet when compared to the wild type control animals. These results demonstrated that MINOR overexpression in skeletal muscle resulted in a significant protection from systemic hyperglycemia and insulin resistance.

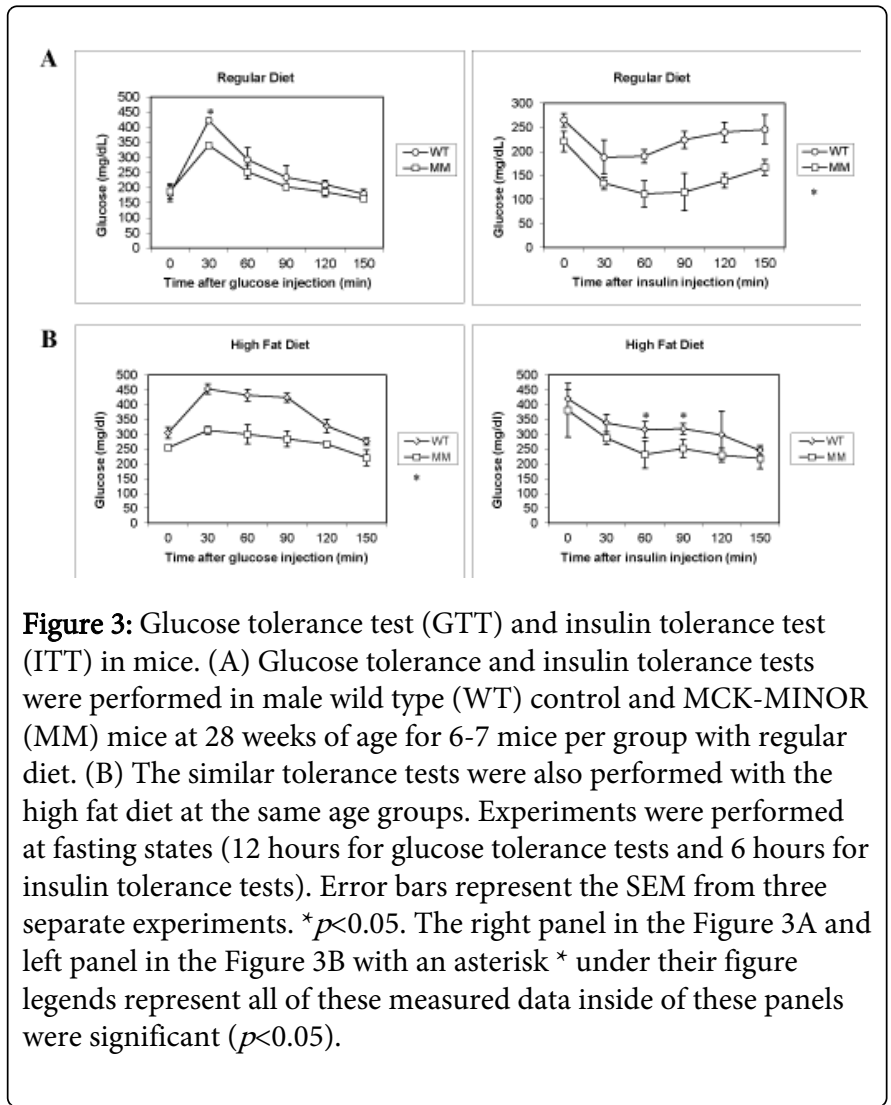

\section{Minor modulates glucose and lipid metabolism in vivo}

As one of the primary target tissues for insulin action, skeletal muscle is the main site of insulin stimulated glucose uptake [13,14]. To determine the role of MINOR in glucose homeostasis, we assessed insulin stimulated glucose transport activity in both skeletal muscle tissues of MCK-MINOR and wild type mice either on regular diet or high fat diet since glucose transport is the rate-limiting step in insulin's ability to stimulate glucose uptake and metabolism (Figure 4). Our results indicated that insulin augmented glucose uptakes in MCKMINOR mice were averagely increased by $38 \%(p<0.05)$ on regular diet and $20 \%(\mathrm{p}<0.01)$ on high fat diet compared to their wild type controls. These results indicated that MINOR can significantly enhance insulin stimulated glucose transport capacity in skeletal muscle, suggesting a novel role of MINOR in improving insulin sensitivity in this target tissue. 


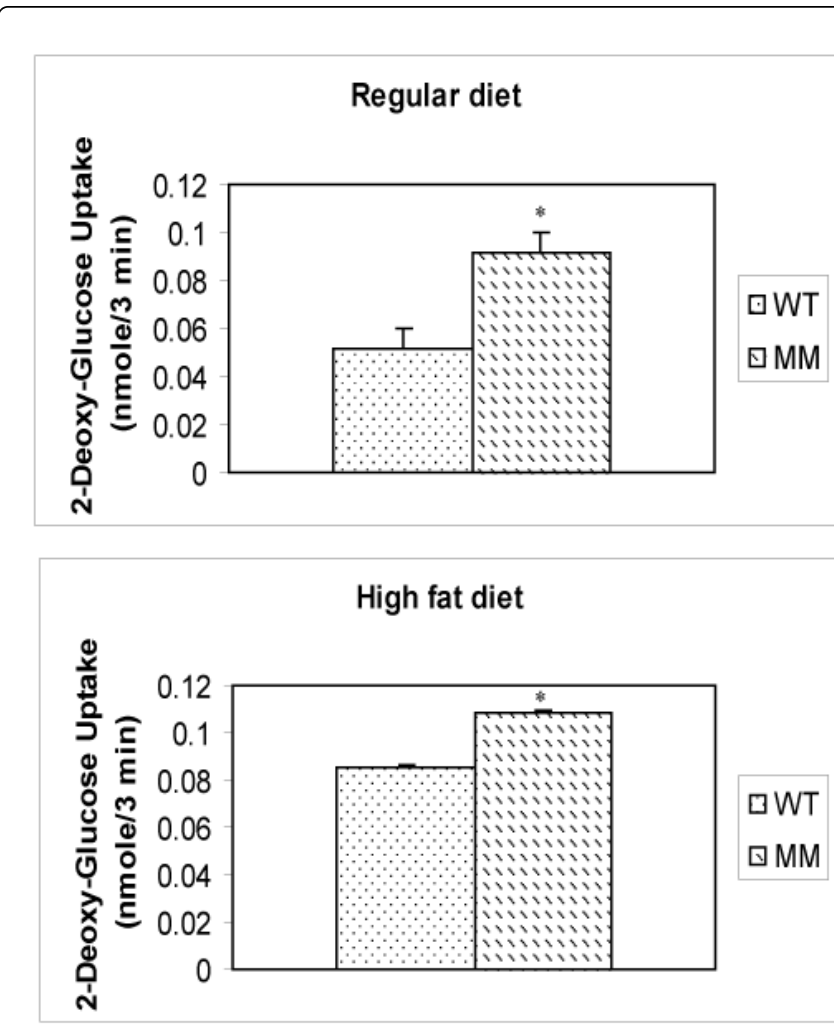

Figure 4: MINOR modulates glucose and lipid metabolism in vivo. Glucose uptake assays in soleus muscle strips which sampled from control wild type (WT) or MCK-MINOR (MM) transgenic mice at 16 weeks of age under regular diet or high fat diet conditions were performed as previously described [13]. For measurement of glucose transport activity, the muscle strips were treaded with $1 \mathrm{~N}$ $\mathrm{NaOH}$ at $70^{\circ} \mathrm{C}$ for $5 \mathrm{~min}$; the aliquots of the supernatant were centrifuged and added to the scintillation mixtures and then counted for the isotope activities. Results represented the mean \pm SE from three separate experiments with 5-6 mice per group. $p<0.05$.

\section{Enhanced insulin signaling pathways in transgenic mice}

We next asked whether the MINOR enhanced insulin action in skeletal muscle alters specific molecules in insulin signaling pathway. To investigate this question, we examined the insulin stimulated phosphorylation levels of $\mathrm{Akt}^{\mathrm{Ser} 473}$, AMPK ${ }^{\text {Thr172 }}$, GSK-3Ser21/Ser9 and expression of IRS-1 and Glut4 in soleus skeletal muscle tissues from the MCK-MINOR and control wild type mice on both regular diet and high fat diet (Figure 5). Our data showed that all of these insulin simulated phosphorylation levels in skeletal muscle of MCK-MINOR mice were highly favorable for insulin action than their controls on either regular diet or high fat diet. In addition, we also observed higher expression levels of IRS-1 and Glut4 in skeletal muscle of the MCKMINOR transgenic mice than those from their wild type control animals.

\section{Reduction of lipid accumulation in transgenic mice}

Since altered adiposity and increased tissue insulin sensitivity often influence lipid profiles in vivo $[15,16]$, we wondered whether lipid levels in plasma from these transgenic animals will beaffected due to the MINOR overexpression in skeletal muscle. Our results demonstrated that the levels of triglyceride, cholesterol and free fatty acids in plasma from MCK-MINOR transgenic mice were significantly lower than those from the wild type control animals (Figure 6). We reasoned that this lipid environment would be favorable to insulin action in vivo.

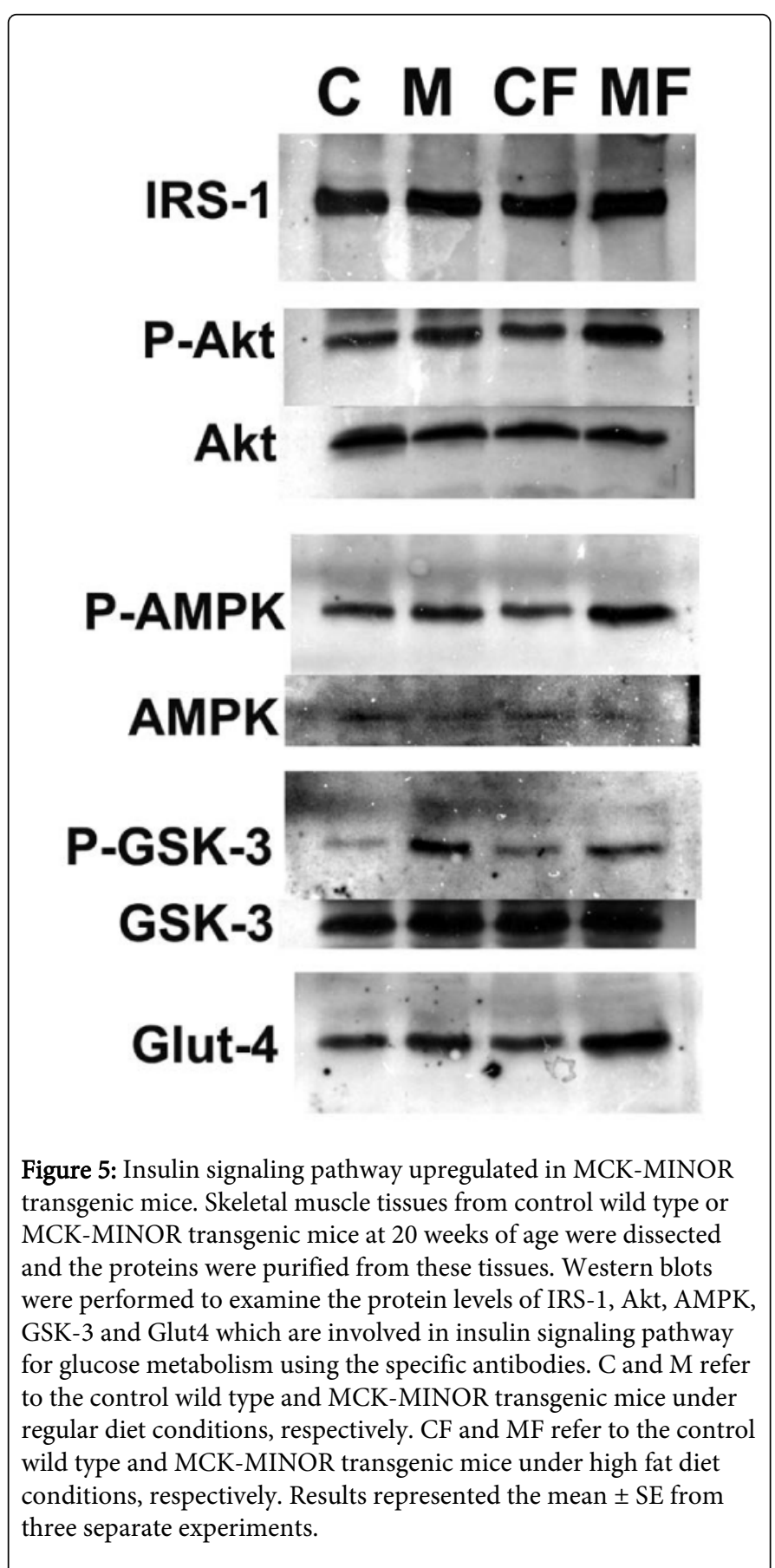




\section{Discussion}

The present results demonstrate for the first time that the MINOR overexpression in skeletal muscle results a reduction of body weight with reduced of fat deposit; these transgenic mice have significant improved insulin sensitivity and glucose tolerance in addition of reduced plasma levels of triglyceride, cholesterol and free fatty acids. Moreover, MINOR overexpression increased insulin-mediated phosphorylations of Akt, AMPK, and GSK-3, which are critical components of insulin signal pathway. These data implicated a role for MINOR in the modulation of insulin action and in the pathogenesis of insulin resistance.
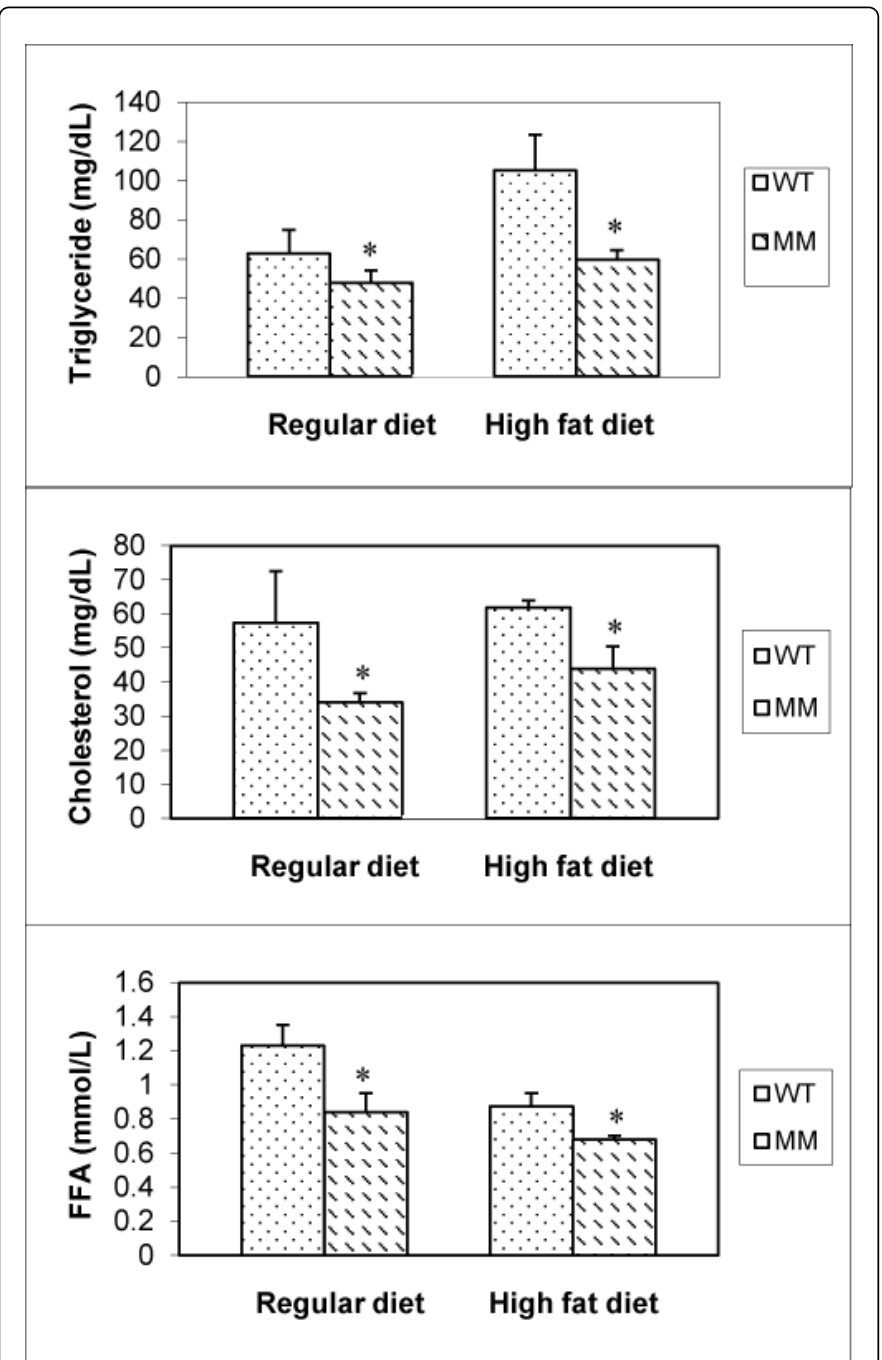

Figure 6: Decreases of the plasma lipid levels in MCK-MINOR transgenic mice. Lipid levels of triglyceride, cholesterol and free fatty acids in plasmas from control wild type (WT) or MCKMINOR transgenic mice at 25 weeks of age under regular diet or high fat diet conditions were measured as previously described [12]. All of these results represented the mean \pm SE from three separate experiments with 5-6 mice per group, $p<0.05$.

Numerous studies have identified NR4A orphan nuclear receptors as regulators for metabolic function in a cell- and tissue-specific manner [17]. Skeletal muscle is one of important target tissues for insulin function and also is a major tissue for lipid and glucose metabolism in vivo. Previous studies have shown that Nur77 (NR4A1), another member of the NR4A orphan nuclear receptors, knockout mice exhibited metabolic changes under high fat diet condition $[18,19]$. These Nur77 knockout mice showed increased weight gain, insulin resistance in skeletal muscle, and slower blood glucose clearance with decreased expression of Glut4 gene, suggesting that Nur77 receptor play an important role in regulating the expression of metabolic genes specifically involved in glucose transport, insulin signaling. Conversely, our MINOR skeletal muscle tissue specific transgenic mice presented very similar results with reduced body weight, improved insulin and glucose intolerances, reduced plasma levels of triglyceride, cholesterol and free fatty acid as well as enhanced expression of genes which are related to insulin action and its signaling pathways. Interestingly, in these Nur77 knockout mice there were the dramatic compensatory increases in MINOR expression detected $[20,21]$, however, we have not observed detectable changes of Nur77 or Nurr1 genes in the MINOR transgenic mice when compared to WT mice, probably due to the differences between global knockout and tissue specific overexpression. Whichever, these studies have indicated that expression and function of MINOR and Nur77 nuclear receptors are strongly associated with metabolic activities in skeletal muscle.

In summary, we have first time found that MINOR overexpression in skeletal muscle results a reduction of body weight with reduced of fat deposit; these transgenic mice have significant improved insulin sensitivity and glucose tolerance in addition of reduced plasma levels of triglyceride, cholesterol and free fatty acids. MINOR also enhances some key components ofinsulin signaling pathway for insulin action in skeletal muscle. Thus, MINOR as a novel insulin enhancer to function in skeletal muscle increases insulin action and energy expenditure process in vivo.

\section{Acknowledgments}

We are graceful to Drs. Morris Birnbaum (University of Pennsylvania) and Ron Kahn (Harvard) for kindly providing the MCK promoter plasmid for our experiments. This work was supported by grants from the National Institutes of Health (DK-38765; PO1 HL-55782) to T.G., and by grants from the American Diabetes Association (1-07-RA-49; 1-13-IN-19) to Y.F.

\section{References}

1. Wu X, Wang J, Cui X, Maianu L, Rhees B, et al. (2007) The effect of insulin on expression of genes and biochemical pathways in human skeletal muscle. Endocrine 31: 5-17.

2. Ohkura N, Hijikuro M, Yamamoto A, Miki K (1994) Molecular cloning of a novel thyroid/steroid receptor superfamily gene from cultured rat neuronal cells. Biochem Biophys Res Commun 205: 1959-1965.

3. Milbrandt J (1988) Nerve growth factor induces a gene homologous to the glucocorticoid receptor gene. Neuron 1: 183-188.

4. Hazel TG, Nathans D, Lau LF (1988) A gene inducible by serum growth factors encodes a member of the steroid and thyroid hormone receptor superfamily. ProcNatlAcadSci U S A 85: 8444-8448.

5. Scearce LM, Laz TM, Hazel TG, Lau LF, Taub R (1993) RNR-1, a nuclear receptor in the NGFI-B/Nur77 family that is rapidly induced in regenerating liver. J BiolChem 268: 8855-8861.

6. Hedvat CV, Irving SG (1995) The isolation and characterization of MINOR, a novel mitogen-inducible nuclear orphan receptor. MolEndocrinol 9: 1692-1700. 
Citation: Zhang W, Garvey JW, Nanlan Luo N, Garvey WT, Fu Y (2014) MINOR (NR4A3) Overexpression in Mouse Skeletal MuscleEnhances Insulin Action. J Mol Genet Med S1: 021. doi:10.4172/1747-0862.S1-021

Page 6 of 6

7. Pei L, Castrillo A, Chen M, Hoffmann A, Tontonoz P (2005) Induction of NR4A orphan nuclear receptor expression in macrophages in response to inflammatory stimuli. J BiolChem 280: 29256-29262.

8. Martínez-González J, Badimon L (2005) The NR4A subfamily of nuclear receptors: new early genes regulated by growth factors in vascular cells. Cardiovasc Res 65: 609-618.

9. Zhu X, Walton RG, Tian L, Luo N, Ho SR, et al. (2013) Prostaglandin A2 enhances cellular insulin sensitivity via a mechanism that involves the orphan nuclear receptor NR4A3. HormMetab Res 45: 213-220.

10. Fu Y, Luo L, Luo N, Zhu X, Garvey WT (2007) NR4A orphan nuclear receptors modulate insulin action and the glucose transport system: potential role in insulin resistance. J BiolChem 282: 31525-31533.

11. Maeda K, Cao H, Kono K, Gorgun CZ, Furuhashi M, et al. (2005) Adipocyte/macrophage fatty acid binding proteins control integrated metabolic responses in obesity and diabetes. Cell Metab 1: 107-119.

12. Wredenberg A, Freyer C, Sandström ME, Katz A, Wibom R, et al. (2006) Respiratory chain dysfunction in skeletal muscle does not cause insulin resistance. Biochem Biophys Res Commun 350: 202-207.

13. Zisman A, Peroni OD, Abel ED, Michael MD, Mauvais-Jarvis F, et al (2000) Targeted disruption of the glucose transporter 4 selectively in muscle causes insulin resistance and glucose intolerance. Nat Med 6: 924-928.

14. Garvey WT, Maianu L, Zhu JH, Brechtel-Hook G, Wallace P, et al. (1998) Evidence for defects in the trafficking and translocation of GLUT4 glucose transporters in skeletal muscle as a cause of human insulin resistance. J Clin Invest 101: 2377-2386.
15. Abel ED, Peroni O, Kim JK, Kim YB, Boss O, et al. (2001) Adiposeselective targeting of the GLUT4 gene impairs insulin action in muscle and liver. Nature 409: 729-733.

16. Lara-Castro C, Fu Y, Chung BH, Garvey WT (2007) Adiponectin and the metabolic syndrome: mechanisms mediating risk for metabolic and cardiovascular disease. CurrOpinLipidol 18: 263-270.

17. Pearen MA, Muscat GE (2010) Minireview: Nuclear hormone receptor 4A signaling: implications for metabolic disease. MolEndocrinol 24: 1891-1903.

18. Chao LC, Zhang Z, Pei L, Saito T, Tontonoz P, et al. (2007) Nur77 coordinately regulates expression of genes linked to glucose metabolism in skeletal muscle. MolEndocrinol 21: 2152-2163.

19. Chao LC, Wroblewski K, Zhang Z, Pei L, Vergnes L, et al. (2009) Insulin resistance and altered systemic glucose metabolism in mice lacking Nur77. Diabetes 58: 2788-2796.

20. Fernandez PM, Brunel F, Jimenez MA, Saez JM, Cereghini S, et al. (2000) Nuclear receptors Nor1 and NGFI-B/Nur77 play similar, albeit distinct, roles in the hypothalamo-pituitary-adrenal axis. Endocrinology 141: 2392-2400.

21. Kanzleiter T, Schneider T, Walter I, Bolze F, Eickhorst C, et al. (2005) Evidence for $\mathrm{Nr} 4 \mathrm{a} 1$ as a cold-induced effector of brown fat thermogenesis. Physiol Genomics 24: 37-44.
This article was originally published in a special issue, entitled: "Molecular \&

Cellular Aspects in Obesity and Diabetes", Edited by Dr. Masayoshi

Yamaguchi, Emory University School of Medicine, USA 